\title{
INTRA-CELLULAR STAPHYLOCOCCUS AUREUS ALONE CAUSES INFECTION IN VIVO
}

\author{
Therwa Hamza ${ }^{1,2}$, Matthew Dietz ${ }^{1}$, Danh Pham ${ }^{1}$, Nina Clovis ${ }^{1}$, Suzanne Danley ${ }^{1}$ and Bingyun $\operatorname{Li}^{1,2,3,4, *}$ \\ ${ }^{1}$ Department of Orthopaedics, School of Medicine, West Virginia University, Morgantown, WV 26506, USA \\ ${ }^{2}$ Department of Basic Pharmaceutical Sciences, School of Pharmacy, West Virginia University, Morgantown, \\ WV 26506, USA \\ ${ }^{3}$ WVNano Initiative, Morgantown, WV 26506, USA \\ ${ }^{4}$ Mary Babb Randolph Cancer Center, Morgantown, WV 26506, USA
}

\begin{abstract}
Chronic and recurrent bone infections occur frequently but have not been explained. Staphylococcus aureus (S. aureus) is often found among chronic and recurrent infections and may be responsible for such infections. One possible reason is that $S$. aureus can internalize and survive within host cells and by doing so, $S$. aureus can evade both host defense mechanisms and most conventional antibiotic treatments. In this study, we hypothesized that intra-cellular S. aureus could induce infections in vivo. Osteoblasts were infected with $S$. aureus and, after eliminating extra-cellular $S$. aureus, inoculated into an open fracture rat model. Bacterial cultures and radiographic observations at post-operative day 21 confirmed local bone infections in animals inoculated with intra-cellular S. aureus within osteoblasts alone. We present direct in vivo evidence that intra-cellular $S$. aureus could be sufficient to induce bone infection in animals; we found that intra-cellular $S$. aureus inoculation of as low as $10^{2}$ colony forming units could induce severe bone infections. Our data may suggest that intra-cellular S. aureus can "hide" in host cells during symptom-free periods and, under certain conditions, they may escape and lead to infection recurrence. Intra-cellular $S$. aureus therefore could play an important role in the pathogenesis of $S$. aureus infections, especially those chronic and recurrent infections in which disease episodes may be separated by weeks, months, or even years.
\end{abstract}

Keywords: Chronic infection; infection recurrence; intracellular pathogen; Staphylococcus aureus; in vivo.

*Address for correspondence:

Bingyun Li, PhD

Nanomedicine Laboratory

Department of Orthopaedics

School of Medicine, West Virginia University

Morgantown, WV 26506-9196, USA

Telephone Number: 1-304-293-1075

FAX Number: 1-304-293-7070

E-mail: bili@hsc.wvu.edu
Introduction

Bone infection or osteomyelitis is a significant clinical problem that can lead to prolonged hospitalization, sepsis, poor functional outcome, and even death (Sugarman and Young, 1989; Johnson et al., 2007; de Lissovoy et al., 2009; Pull ter Gunne and Cohen, 2009). Patients with traumatic open fractures have a high risk of infection (5-33\%) due to microbial contamination and soft tissue damage (Zalavras and Patzakis, 2003); such infections are often hard to eradicate, chronic, and recurrent (Berendt and Byren, 2004). For instance, over a million Americans are hospitalized every year for bone fractures (Canale, 2003); such injuries are increasingly common because of increased survivability of high energy trauma in civilian settings as well as in combat activities. Treatment of bone infections, particularly chronic and recurrent infections, frequently requires long-term hospitalization for repeated debridements and prolonged parenteral antibiotic therapy. Bacteria including Staphylococcus aureus (S. aureus), however, are often surprisingly successful in overcoming both host defense mechanisms and antibiotic treatments and result in chronic and recurrent bone infections. Indeed, a high rate of recurrence of bone infections was reported where $\sim 17 \%$ of infections related to traumatic extremity injuries recur (Murray et al., 2008; Murray et al., 2011). One approach that bacteria may use to elude host defenses and antibiotics is to "hide" themselves intra-cellularly within host cells (Ciampolini and Harding, 2000).

A wide range of bacterial species related to bone infections has been isolated. The pathogen $S$. aureus is one of the most common causative agents of bone infections (Sax and Lew, 1999). S. aureus commonly colonizes the skin, skin glands, and mucous membranes, specifically in the noses of healthy individuals. $S$. aureus expresses a large number of cell surface proteins (i.e. adhesins) of the microbial surface components that recognize adhesive matrix molecules or MSCRAMMs (Patti and Höök, 1994; Clarke and Foster, 2006) by which it can colonize the bone matrix and adhere to bone cell surfaces. S. aureus, while generally considered an extra-cellular pathogen, is one such bacterium that has the ability to invade and live within different cell types, both phagocytic and nonphagoytic cells (reviewed by Garzoni and Kelley, 2009). By "hiding" inside the host cells, S. aureus may elude host defenses and most antibiotic treatments, and may be responsible for chronic and recurrent bone infections.

The objective of this study was to determine whether intra-cellular $S$. aureus can induce bone infections in a rat model. By pre-infecting osteoblasts in vitro with $S$. 


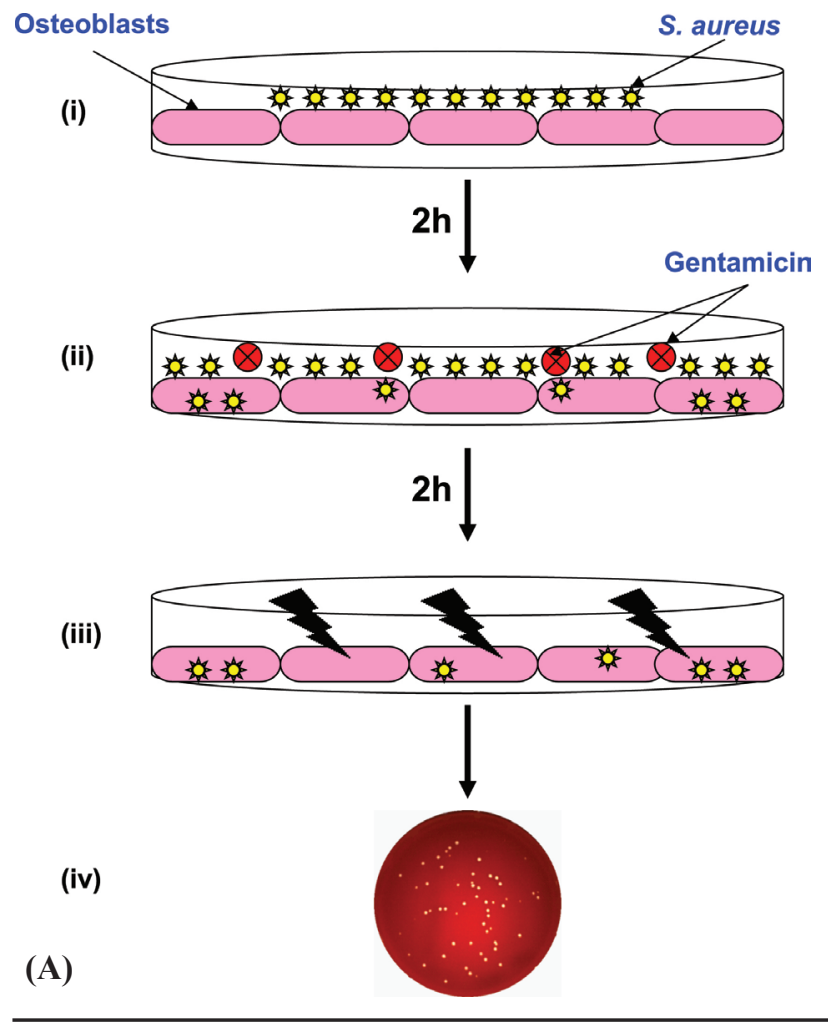

aureus, killing extra-cellular bacteria with gentamicin, and inoculating infected osteoblasts into an open fracture rat model, we examined bone infections in rats induced by intra-cellular $S$. aureus.

\section{Materials and Methods}

\section{Reagents}

Dulbecco's modified Eagle's medium/F-12 (DMEM/F-12) and phosphate buffered saline (PBS) were purchased from Lonza (Walkersville, MD, USA). Tryptic soy broth (TSB) and tryptic soy agar (TSA w/5 \% sheep blood) were from Fisher Scientific (Pittsburgh, PA, USA). Fetal bovine serum (FBS), $0.25 \%$ trypsin-ethylenediaminetetraacetic acid (EDTA), and a solution of penicillin $(100 \mathrm{U} / \mathrm{mL}) /$ streptomycin $(100 \mathrm{mg} / \mathrm{mL})$ were obtained from Invitrogen (Carlsbad, CA, USA). Triton X-100 was from SigmaAldrich (St. Louis, MO, USA). S. aureus (ATCC 25923) and rat osteoblast cells (UMR-106) were purchased from American Type Culture Collection (ATCC, Manassas, VA, USA). Susceptibility tests showed that the $S$. aureus strain was susceptible to cefazolin, cefoxitin, ciprofloxacin, clindamycin, erythromycin, gentamicin, levofloxacin, linezolid, moxifloxacin, oxacillin, penicillin, rifampin, tetracycline, tigecycline, and vancomycin and was resistant to ampicillin.

\section{Preparation of osteoblasts with intra-cellular $S$. aureus}

Rat osteoblast cells were seeded at a density of $5 \times 10^{5}$ osteoblasts $/ \mathrm{mL}$ and cultured in 6-well plates overnight in DMEM/F-12 supplemented with $10 \%$ FBS and $1 \%$ penicillin/streptomycin solution to form a monolayer. Fresh $S$. aureus inoculum was prepared by suspending 5 colonies

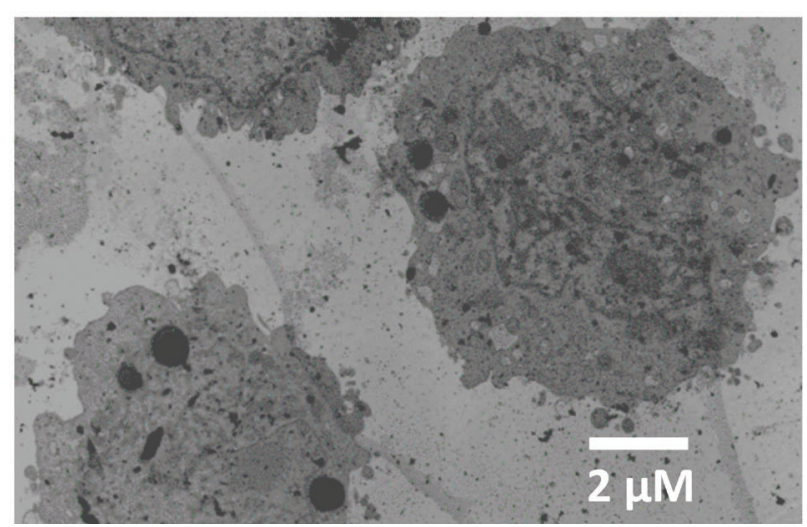

(B)

Fig. 1. (A) A schematic diagram of the infection model of osteoblasts and S. aureus: (i) Rat osteoblasts were infected with $S$. aureus for 2 h. (ii) Extra-cellular $S$. aureus were killed with gentamicin. (iii) Osteoblasts were lysed using a lysing buffer. (iv) Cell lysates were cultured on blood agar plates to count live intra-cellular $S$. aureus colonies. (B) TEM imaging of intra-cellular $S$. aureus within osteoblasts. of $S$. aureus into $5 \mathrm{~mL}$ TSB and incubating at $37{ }^{\circ} \mathrm{C}$ for $18 \mathrm{~h}$. The bacterial suspension was centrifuged (3,750 rpm) at $4{ }^{\circ} \mathrm{C}$ for $15 \mathrm{~min}$ and washed once with PBS; its optical density was adjusted to $5 \times 10^{8}$ colony forming units $/ \mathrm{mL}$ (CFU/mL) of $S$. aureus. Rat osteoblasts and $S$. aureus were then cultured as follows (Fig. 1A): (i) The osteoblast monolayer was washed twice with PBS. S. aureus was pelleted, re-suspended in DMEM/F-12 (without FBS and antibiotics), and then added to the osteoblast monolayer at a multiplicity of infection (MOI) of 500:1 (S. aureus to osteoblasts). Osteoblasts and $S$. aureus were next cultured together at $37{ }^{\circ} \mathrm{C}$ in a $5 \% \mathrm{CO}_{2}$ humidified incubator for $2 \mathrm{~h}$. (ii) The osteoblast monolayer was washed twice with PBS to remove any non-adherent bacteria, and cell culture media supplemented with gentamicin $(100 \mu \mathrm{g} / \mathrm{mL})$ was added and kept for $2 \mathrm{~h}$ to kill any extra-cellular bacteria. Gentamicin treatment is commonly used to eliminate extra-cellular bacteria (Wright and Friedland, 2002; Jevon, et al., 1999); gentamicin is unable to penetrate mammalian cell membranes within short time periods (Plotkowski et al., 1999; Hamad et al., 2010). After gentamicin treatment, osteoblasts were detached and viable osteoblasts were counted using a hemocytometer (BrightLine Hausser Scientific, Horsham, PA, USA) following the trypan-blue exclusion assay; the viability of osteoblasts was approximately $94.2 \pm 5.5 \%$. Infected osteoblasts were immediately used; intra-cellular $S$. aureus within osteoblasts prior to inoculation into rats was imaged (Fig. 1B). (iii) Samples of infected osteoblasts were lysed using Triton X-100. (iv) Cell lysates were cultured on blood agar plates to determine the CFU that represented the viable colonies of intra-cellular $S$. aureus within osteoblasts. To examine the viability of infected osteoblasts at days postinfection, non-infected (control) and infected osteoblasts were cultured in DMEM/F12 supplemented with $5 \%$ FBS 

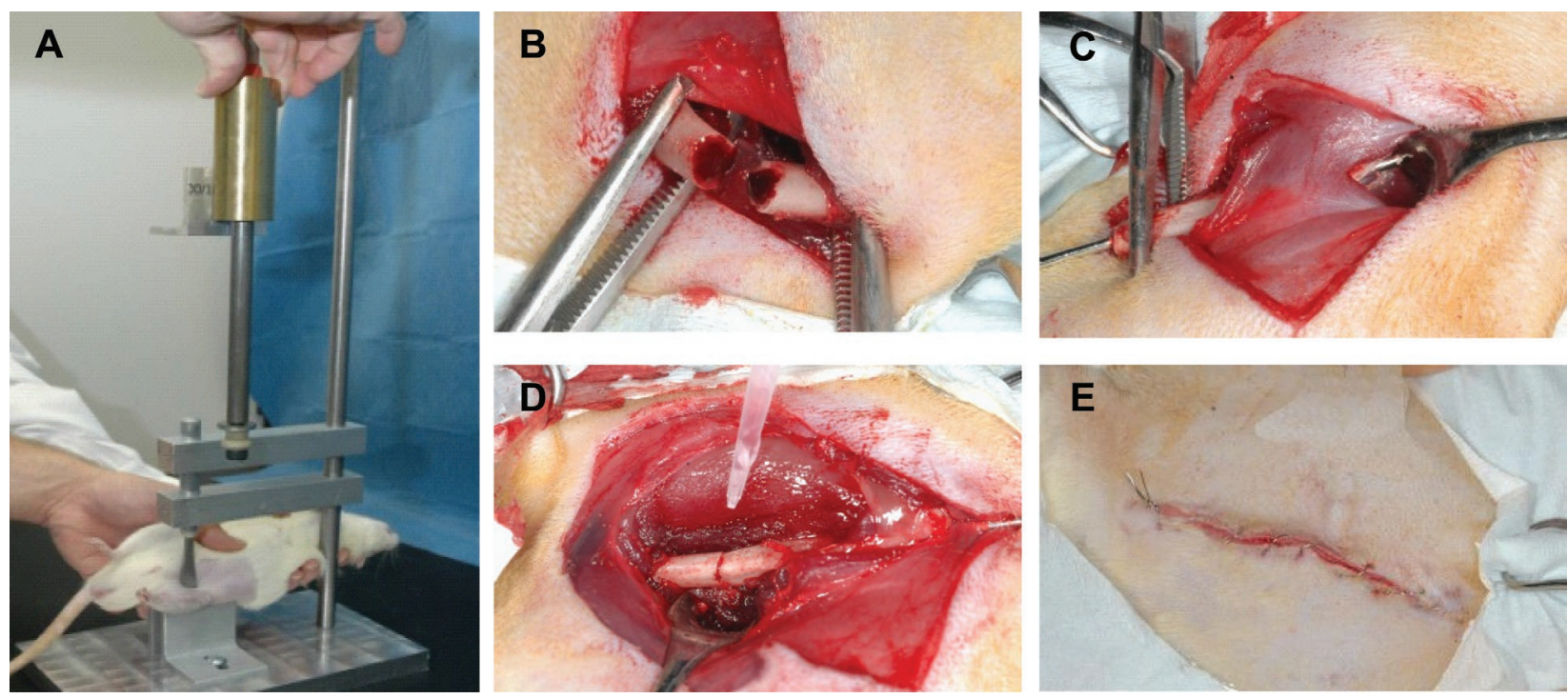

Fig. 2. Surgical procedures: The rat's femur was fractured, fixed with a K-wire, and inoculated with 0.1 mL solution of the assigned inoculum. (A) Creation of a femur fracture using a custom-designed setup. (B) Exposure of the fracture. (C) Intramedullary fixation of the fracture using a K-wire. (D) Inoculation of $0.1 \mathrm{~mL}$ solution using a pipette. (E) Closure of incision.

and $5 \mu \mathrm{g} / \mathrm{mL}$ lysostaphin solution for 1 and $7 \mathrm{~d}$; media was changed every $3 \mathrm{~d}$ for the day 7 samples. Lysostaphin does not penetrate mammalian cell membranes for long time periods, e.g. weeks (Easmon et al., 1978; Kumar, 2008; Maurin and Raoult, 2001). The viability of osteoblasts at post-infection days 1 and 7 was calculated relevant to their control (non-infected) cells. The viability of the infected osteoblasts was found to be $84.6 \pm 6.7 \%$ and $77.4 \pm 0.6 \%$ at post-infection days 1 and 7, respectively.

\section{Animal groups and open fracture infection procedure}

The study was approved by the West Virginia University Institutional Animal Care and Use Committee. Male Sprague-Dawley rats (400-450 g) were obtained from Hilltop Lab Animals (Scottdale, PA). Our open femur fracture rat model (Li et al., 2009; Boyce et al., 2012) was modified and the surgical procedure is described in brief as follows (Fig. 2): After sedation of the animals with intraperitoneal anesthetic, one hind leg was shaved prior to fracture. A midshaft femur fracture was produced using a custom-made device (Fig. 2A), the surgical site was disinfected, an incision was made to expose the fracture (Fig. 2B), and then the fracture was fixed using an intramedullary stainless steel Kirschner wire or K-wire (Fig. 2C). Next, $100 \mu \mathrm{L}$ of treatment solution was gently pipetted into the fracture site without contacting the surgical instruments (Fig. 2D). Based on the treatment solutions, rats were randomly assigned to one of the following six groups (six rats per group) (Table 1). The incision was carefully closed (Fig. 2E) and post-operative radiographs were taken to verify fracture fixation. All animals were monitored for the study duration of 3 weeks and euthanized at post-operative day 21 . Note that both $10^{2}$ and $10^{6} \mathrm{CFU}$ of extra-cellular $S$. aureus induced bone infections in the same open fracture rat model (Li et al., 2009; Li et al., 2010; Boyce et al., 2012), and a lower extra-cellular $S$. aureus dose of $20 \mathrm{CFU}$, which was expected not to induce infection, was chosen here to evaluate the potential role of intra-cellular $S$. aureus by comparing Group III with Group VI.

\section{Microbiological and radiographic evaluations}

At euthanasia, bone and muscle tissue were collected in $15 \mathrm{~mL}$ centrifuge tubes with $5 \mathrm{~mL}$ PBS and homogenized. Samples of bone and muscle homogenates were diluted and plated on blood agar plates and $S$. aureus colonies were counted after incubating the plates at $37{ }^{\circ} \mathrm{C}$ for $24 \mathrm{~h}$. In addition, K-wires used to fix the fractured femurs were collected at euthanasia, cut into $2 \mathrm{~cm}$-long pieces, rolled on blood agar plates, and cultured at $37^{\circ} \mathrm{C}$ for $24 \mathrm{~h}$. Radiographs were taken at post-operative days 0, 7 and 21.

\section{Body weight and complete blood count}

The body weights of the animals were taken on the day of surgery and immediately before euthanasia; the net weight

Table 1. Animal groups for open fracture infection procedure.

\begin{tabular}{|l|l|}
\hline Group & Treatment solution $(\mathbf{1 0 0} \boldsymbol{\mu} \mathbf{L})$ \\
\hline Group I & PBS \\
\hline Group II & $10^{6}$ osteoblasts \\
\hline Group III & $\begin{array}{l}10^{6} \text { osteoblasts combined with } 20 \text { CFU of extra- } \\
\text { cellular } S \text {. aureus }\end{array}$ \\
\hline Group IV* & $\begin{array}{l}10^{6} \text { osteoblasts with } 10^{2} \text { CFU intra-cellular } S . \\
\text { aureus }\end{array}$ \\
\hline Group V & $\begin{array}{l}10^{6} \text { osteoblasts infected with } 10^{6} \text { CFU intra- } \\
\text { cellular } S \text {. aureus }\end{array}$ \\
\hline Group VI & $\begin{array}{l}10^{6} \text { osteoblasts infected with } 10^{6} \mathrm{CFU} \text { intra- } \\
\text { cellular } S \text {. aureus combined with } 20 \text { CFU of } \\
\text { extra-cellular } S \text {. aureus }\end{array}$ \\
\hline
\end{tabular}

*This solution was obtained by diluting the $10^{6} \mathrm{CFU}$ of intra-cellular S. aureus (i.e. Group V) with non-infected osteoblasts. 
(A)

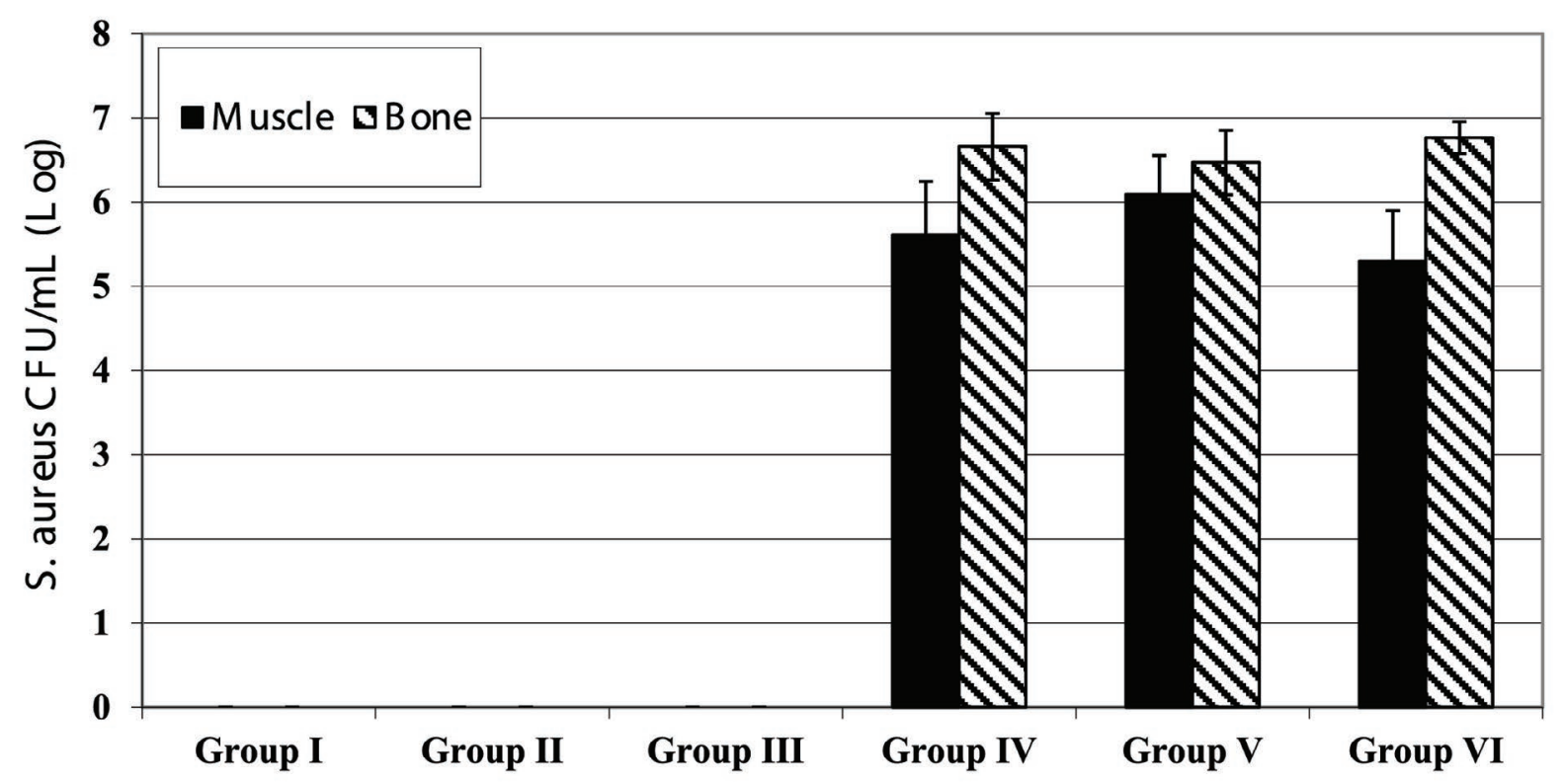

(B)

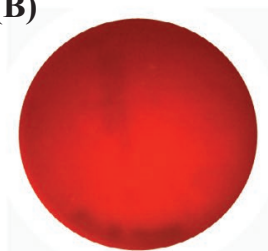

Group I

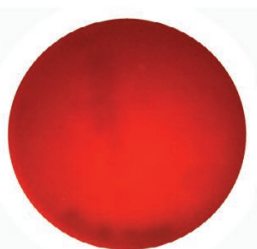

Group II

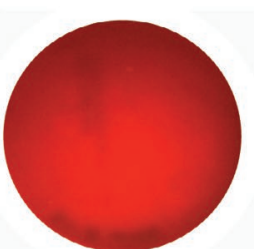

Group III

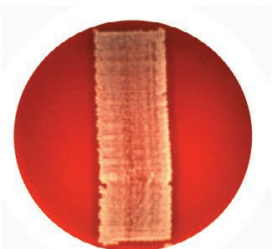

Group IV

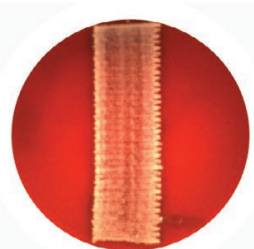

Group V

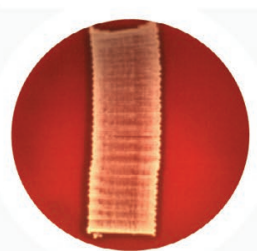

Group VI

Fig. 3. (A) Bone (femur) and muscle tissue homogenates reported high S. aureus CFUs in the animal groups inoculated with infected osteoblasts (i.e. Groups IV, V, and VI); six rats in each group. No CFUs were found in the groups inoculated with PBS, osteoblasts, and a mixture of osteoblasts and 20 CFUs of extra-cellular S. aureus (i.e. Groups I, II, and III). (B) K-wire rolling experiments showed high S. aureus growth in the groups inoculated with infected osteoblasts; no bacterial growth was observed in the groups inoculated with PBS, osteoblasts, and a mixture of osteoblasts and extra-cellular S. aureus (20 CFU). Group I: PBS; Group II: $10^{6}$ osteoblasts; Group III: $10^{6}$ osteoblasts combined with $20 \mathrm{CFU}$ of extra-cellular S. aureus; Group IV: $10^{6}$ osteoblasts with $10^{2} \mathrm{CFU}$ intra-cellular $S$. aureus; Group V: $10^{6}$ osteoblasts infected with $10^{6} \mathrm{CFU}$ intra-cellular S. aureus; Group VI: $10^{6}$ osteoblasts infected with $10^{6}$ CFU intra-cellular $S$. aureus combined with $20 \mathrm{CFU}$ of extra-cellular $S$. aureus.

gain/loss was calculated. Blood samples were collected from the heart immediately prior to euthanasia and analyzed for complete blood counts by Antech Diagnostics, Inc. (Southaven, MS, USA).

\section{Statistical analysis}

Values of CFU, body weight gain/loss, and blood cell counts were expressed as mean \pm standard deviation. Differences in body weight gain/loss and blood cell counts were analyzed using one-way ANOVA. $p$ value $<0.05$ was considered statistically significant. JMP (V9) software was used (SAS Institute, Inc., Cary, NC, USA).

\section{Results}

Intra-cellular $S$. aureus causes high bacterial burden in bone and muscle tissue

Rats (Groups IV, V, and VI) inoculated with infected osteoblasts $\left(10^{2}\right.$ or $10^{6} \mathrm{CFU}$ of intra-cellular $S$. aureus $)$ led to high $\mathrm{CFU}$ counts (i.e. $\sim 10^{6} \mathrm{CFU} / \mathrm{mL}$ ) at post-operative day 21 in the bone and muscle samples (Fig. 3A). There were no significant differences in CFU among the groups (i.e. Groups IV, V, and VI) inoculated with infected osteoblasts at post-operative day 21 , although relatively higher CFU counts were found in the bone samples compared to the muscle samples of the same group. By contrast, no bacteria were observed at post-operative day 21 in the rats (i.e. Groups I, II, and III) inoculated with PBS, osteoblasts, and osteoblasts combined with $20 \mathrm{CFU}$ of extra-cellular S. aureus (Fig. 3A). Correspondingly, heavy bacterial growth was seen in the blood agar plates rolled with the K-wires from animals inoculated with infected osteoblasts $\left(10^{2}\right.$ or $\left.10^{6} \mathrm{CFU}\right)$, while no bacterial colonies were found in $\mathrm{K}$-wires extracted from animals inoculated with PBS, osteoblasts, and osteoblasts combined with $20 \mathrm{CFU}$ of extra-cellular S. aureus (Fig. 3B). Gross observation before euthanasia showed clear pus pockets at the fracture sites in rats inoculated with infected osteoblasts (i.e. Groups IV, V, and VI). No S. aureus growths (data not 
(A)
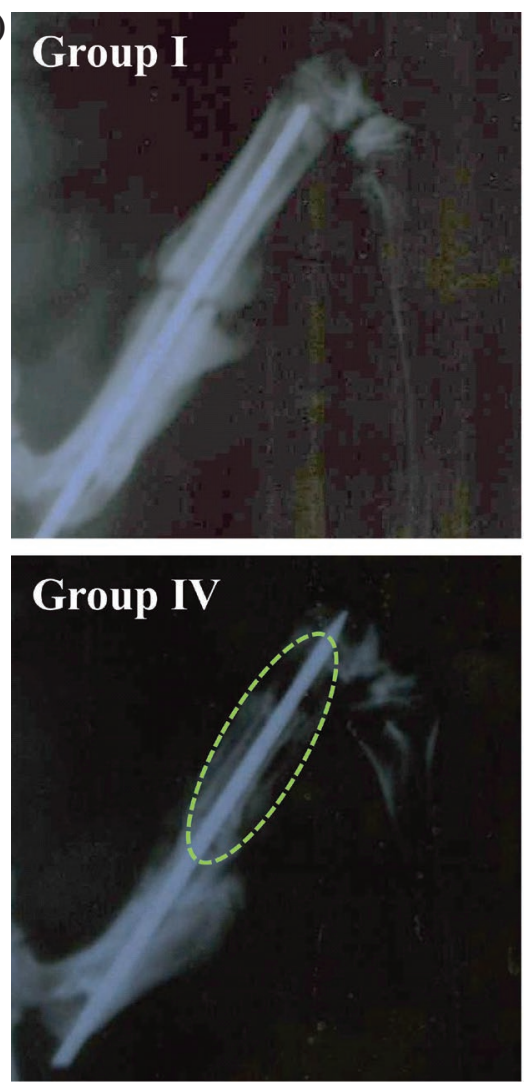

(B)
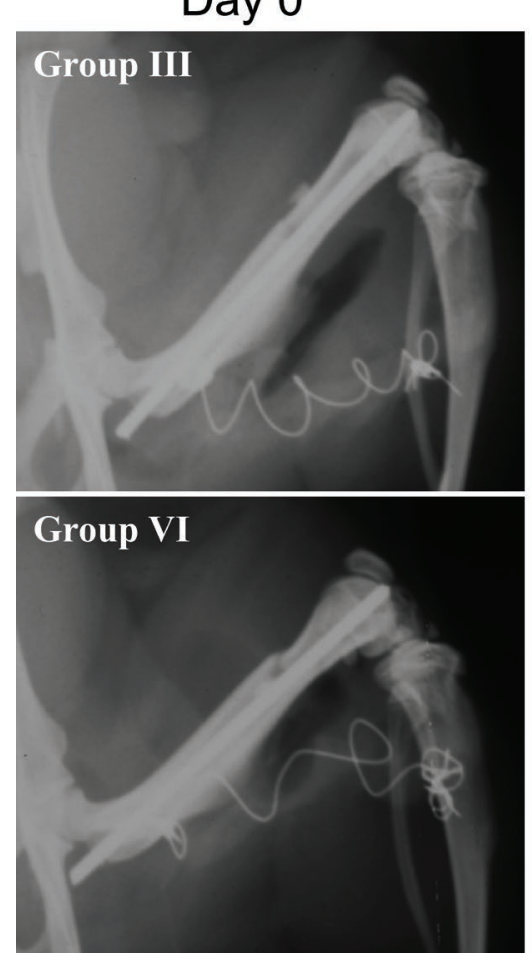
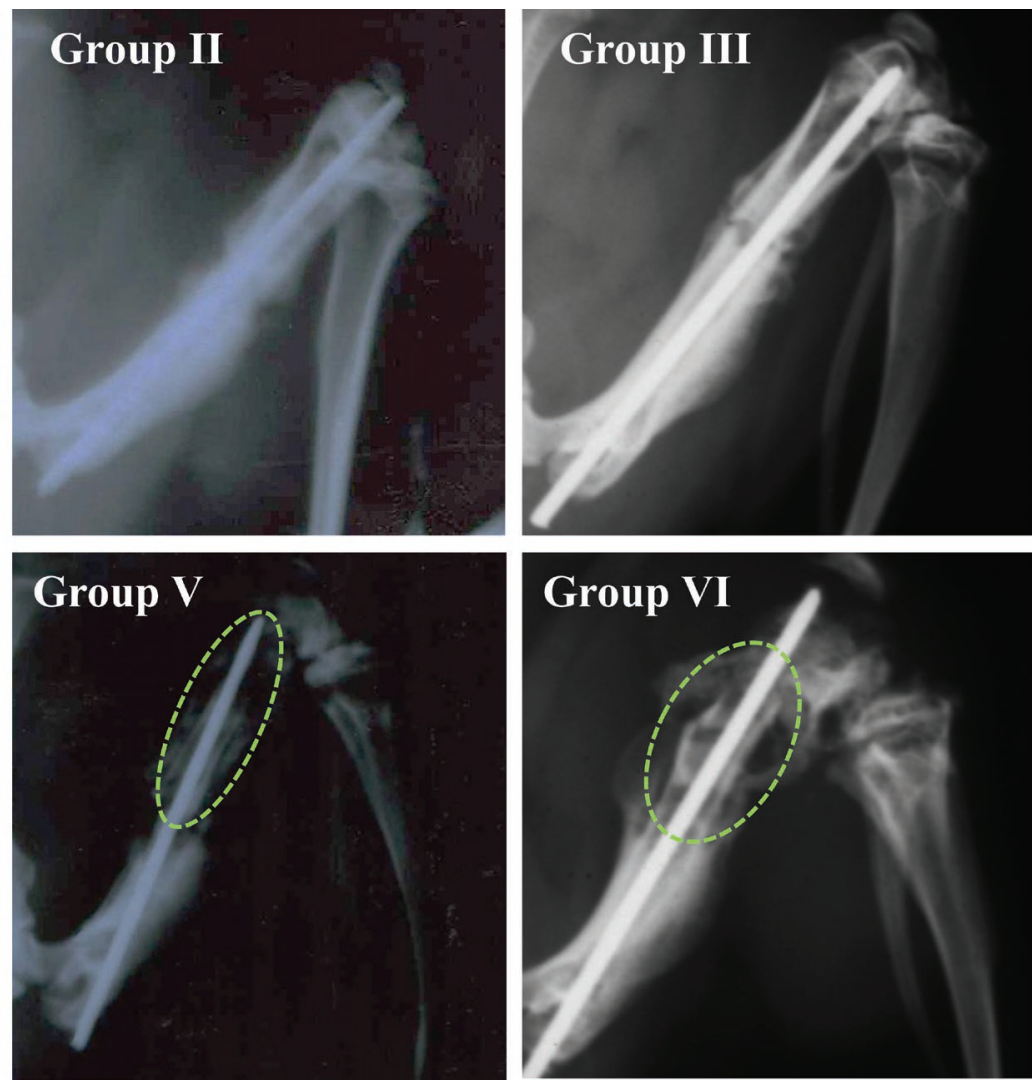

Day 7
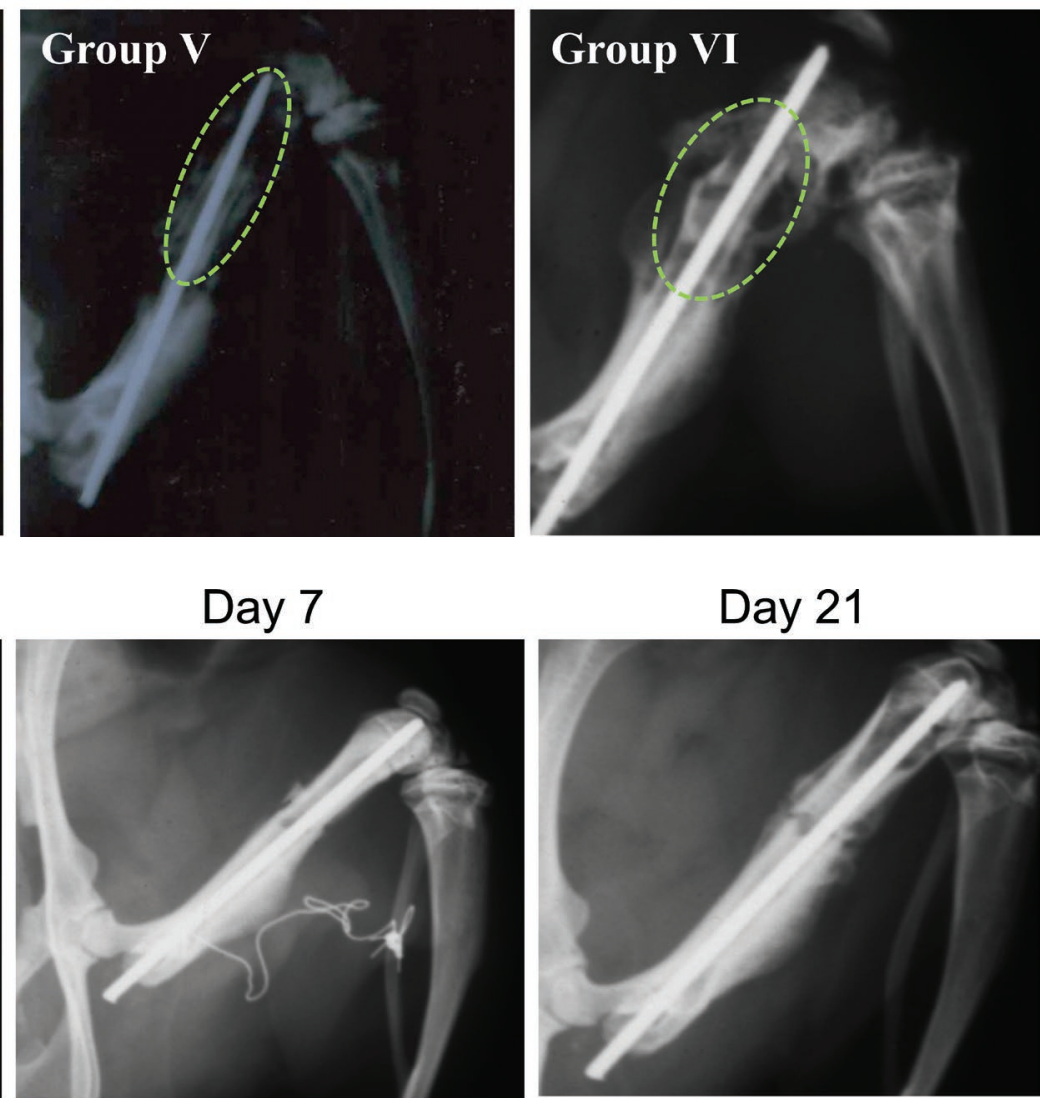

Day 21
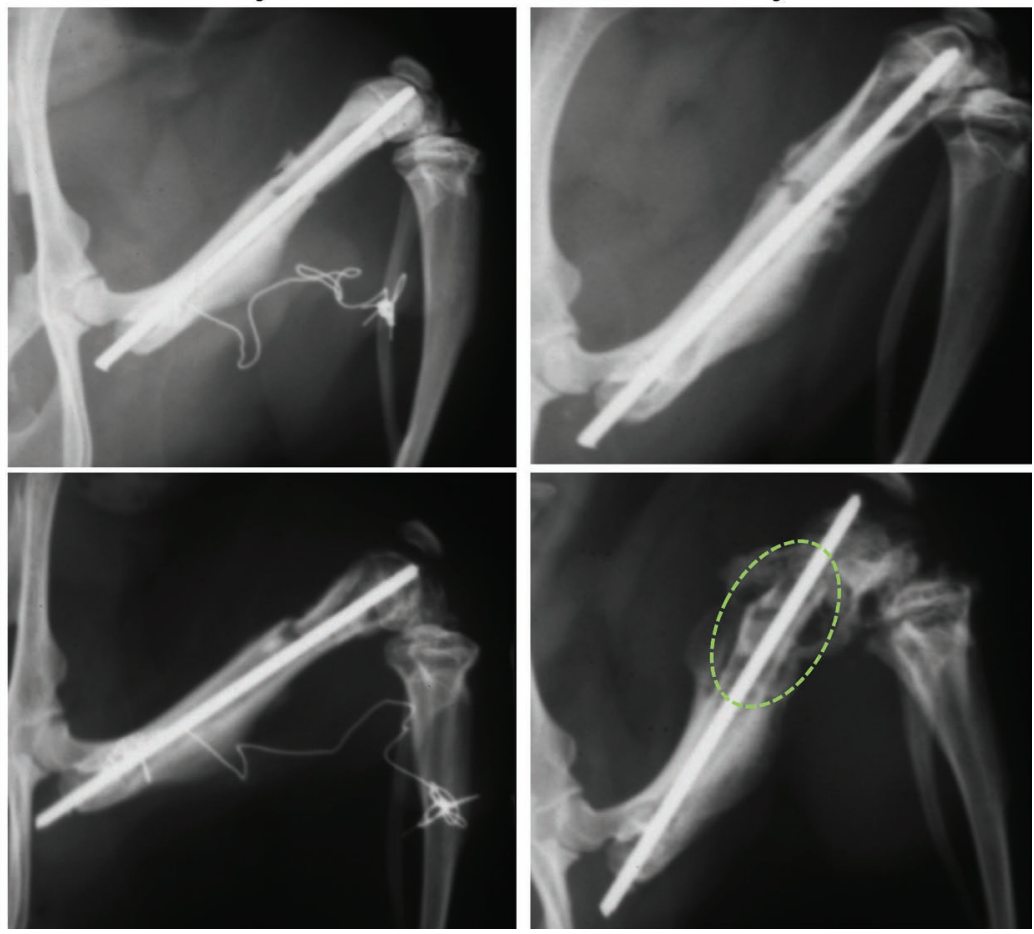

Fig. 4. (A) Radiographs of fractured femurs at post-operative day 21 presented significant osteolysis in the animal groups inoculated with infected osteoblasts (i.e. Groups IV, V, and VI), while normal bone healing was seen in the groups inoculated with PBS, osteoblasts, and a mixture of osteoblasts and extra-cellular S. aureus (20 CFU) (i.e. Groups I, II, and III). (B) Radiographs of fractured femurs at post-operative day 0, 7 and 21 did not show severe osteolysis in the rats inoculated with infected osteoblasts (e.g. Group VI) at post-operative day 7. The dashed circles indicate the areas of significant osteolysis. The curled wires were the stainless steel sutures used to close the incision. Group I: PBS; Group II: $10^{6}$ osteoblasts; Group III: $10^{6}$ osteoblasts combined with $20 \mathrm{CFU}$ of extra-cellular S. aureus; Group IV: $10^{6}$ osteoblasts with $10^{2} \mathrm{CFU}$ intra-cellular S. aureus; Group V: $10^{6}$ osteoblasts infected with $10^{6} \mathrm{CFU}$ intra-cellular S. aureus; Group VI: $10^{6}$ osteoblasts infected with $10^{6} \mathrm{CFU}$ intra-cellular S. aureus combined with 20 CFU of extra-cellular $S$. aureus. 


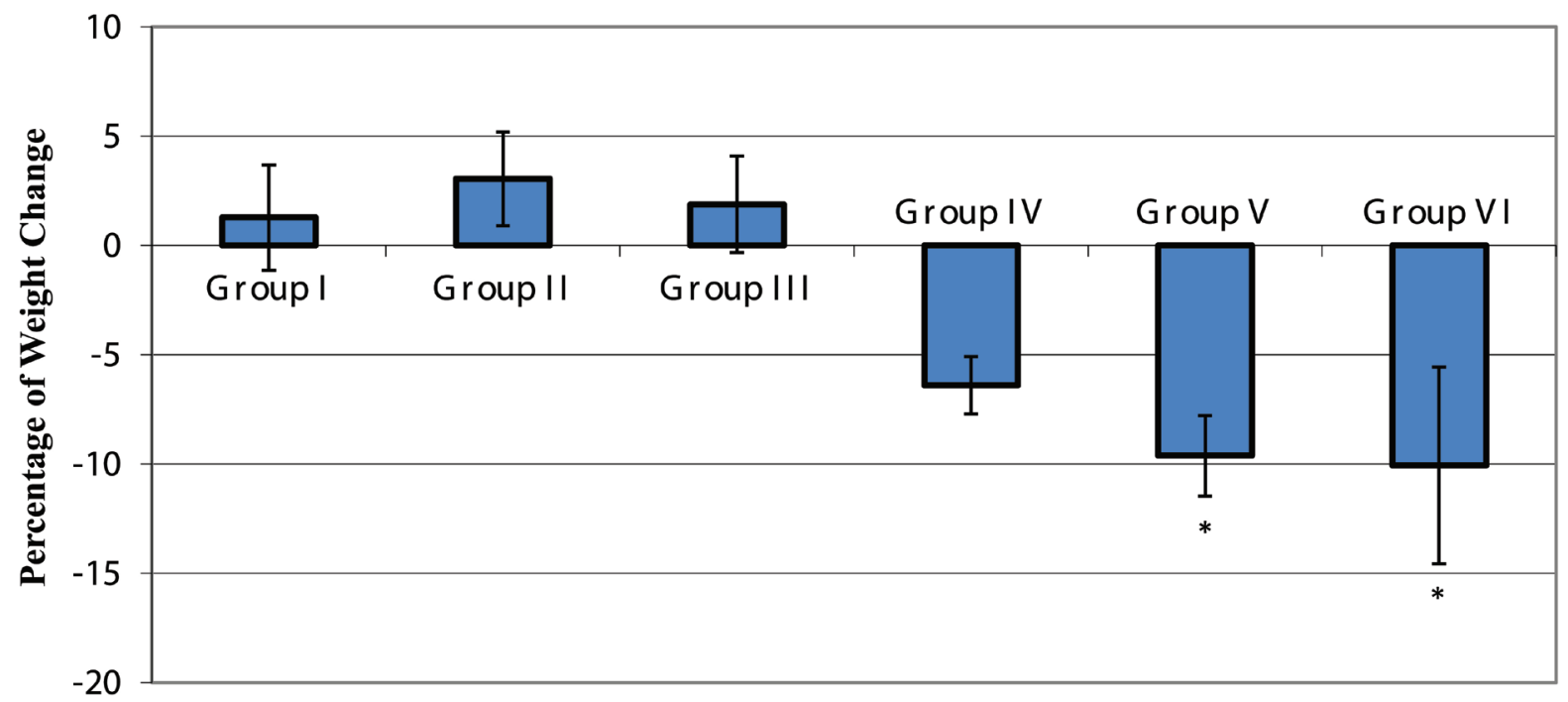

Fig. 5. Net weight loss of the rats at post-operative day 21 was significantly higher in the animal groups inoculated with infected osteoblasts (i.e. Groups V and VI) compared to those in the groups inoculated with PBS, osteoblasts, and a mixture of osteoblasts and $20 \mathrm{CFU}$ of extra-cellular S. aureus (i.e. Groups I, II, and III). Data were an average of six rats in each group. Error bars were standard deviations. Group I: PBS; Group II: $10^{6}$ osteoblasts; Group III: $10^{6}$ osteoblasts combined with $20 \mathrm{CFU}$ of extra-cellular $S$. aureus; Group IV: $10^{6}$ osteoblasts with $10^{2} \mathrm{CFU}$ intracellular S. aureus; Group V: $10^{6}$ osteoblasts infected with $10^{6} \mathrm{CFU}$ intra-cellular S. aureus; Group VI: $10^{6}$ osteoblasts infected with $10^{6} \mathrm{CFU}$ intra-cellular $S$. aureus combined with $20 \mathrm{CFU}$ of extra-cellular $S$. aureus.

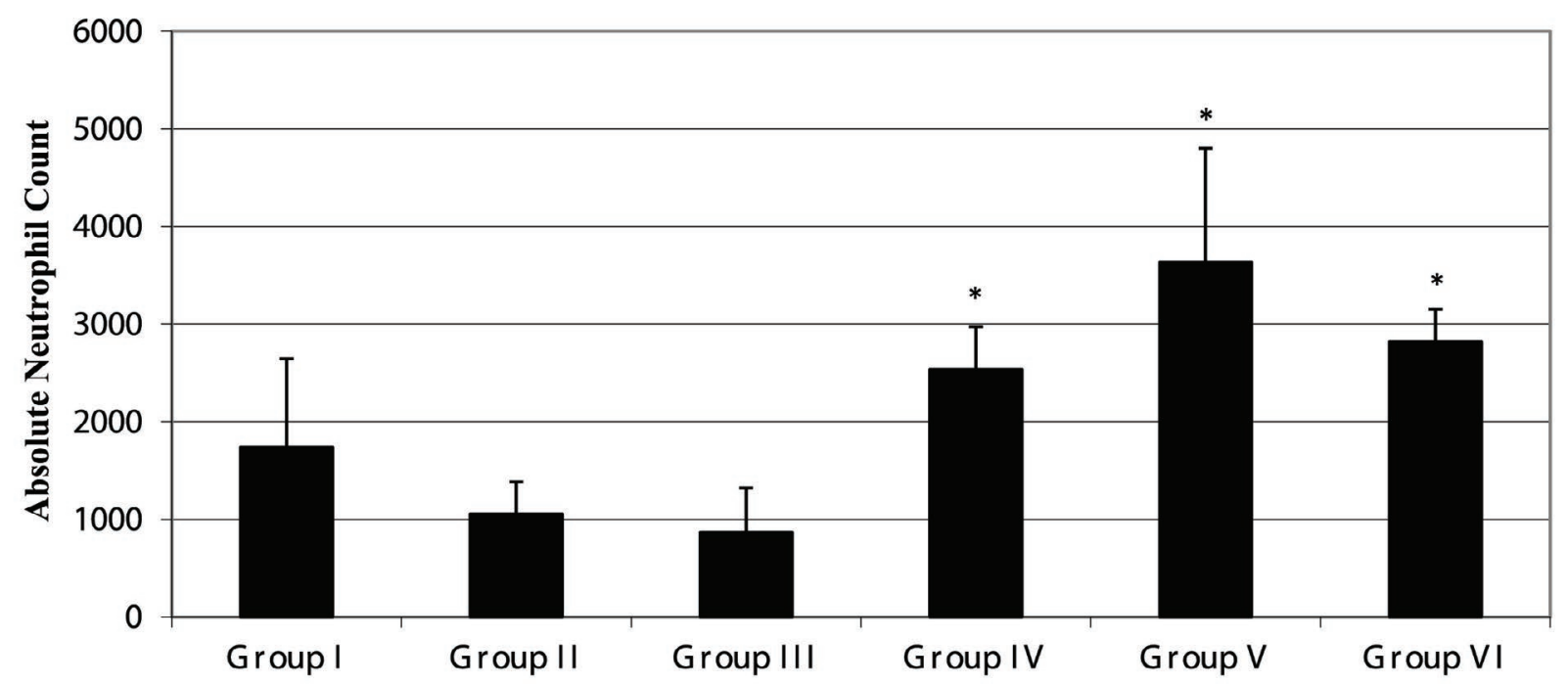

Fig. 6. Complete blood counts at post-operative day 21 showed that neutrophil numbers were significantly higher in the animal groups inoculated with infected osteoblasts (i.e. Groups IV, V, and VI) compared to those groups inoculated with osteoblasts and a mixture of osteoblasts and $20 \mathrm{CFU}$ of extra-cellular S. aureus (i.e. Groups II and III). Error bars were standard deviations. Group I: PBS; Group II: $10^{6}$ osteoblasts; Group III: $10^{6}$ osteoblasts combined with 20 CFU of extra-cellular S. aureus; Group IV: $10^{6}$ osteoblasts with $10^{2}$ CFU intra-cellular S. aureus; Group V: $10^{6}$ osteoblasts infected with $10^{6} \mathrm{CFU}$ intra-cellular $S$. aureus; Group VI: $10^{6}$ osteoblasts infected with $10^{6} \mathrm{CFU}$ intra-cellular S. aureus combined with $20 \mathrm{CFU}$ of extra-cellular S. aureus.

shown) were observed at post-operative day 21 in any of the blood samples.

\section{Radiographic assessment of bone infection}

No signs of infection were observed at post-operative day 21 in the radiographs of rats (i.e. Groups I, II, and III) inoculated with PBS, osteoblasts, and osteoblasts combined with $20 \mathrm{CFU}$ of extra-cellular $S$. aureus (Fig. $4 \mathrm{~A})$. The radiographic images of these animals were characterized by the formation of normal bridging calluses at the fracture sites. By contrast, groups (i.e. Groups IV, $\mathrm{V}$, and VI) inoculated with infected osteoblasts $\left(10^{2}\right.$ or $10^{6}$ CFU) showed severe osteolysis at post-operative day 21 . Meanwhile, no severe osteolysis was observed at postoperative day 7 in the animals inoculated with infected osteoblasts (Fig. 4B); this may indicate that it takes time (more than $7 \mathrm{~d}$ ) to develop infection in this animal model. 


\section{Body weight and complete blood counts}

A significant loss in body weight was observed in rat groups (i.e. Groups V and VI) inoculated with infected osteoblasts of $10^{6} \mathrm{CFU}$ of intra-cellular $S$. aureus and $10^{6} \mathrm{CFU}$ of intracellular $S$. aureus combined with $20 \mathrm{CFU}$ of extra-cellular S. aureus compared to the groups (i.e. Groups I, II, and III) inoculated with PBS, osteoblasts, and osteoblasts combined with $20 \mathrm{CFU}$ of extra-cellular S. aureus (Fig. 5). More weight loss was also observed in Group IV inoculated with infected osteoblasts of $10^{2} \mathrm{CFU}$ of intra-cellular $S$. aureus compared to Groups I, II, and III; however, the differences were not statistically significant (Fig. 5).

The neutrophils in the blood of groups (i.e. Groups IV, $\mathrm{V}$, and VI) inoculated with infected osteoblasts $\left(10^{2}\right.$ or $10^{6}$ CFU) were significantly elevated at post-operative day 21 compared to the groups (i.e. Groups II and III) inoculated with osteoblasts and osteoblasts combined with $20 \mathrm{CFU}$ of extra-cellular S. aureus (Fig. 6). No significant differences (data not shown) were seen at post-operative day 21 in the numbers of lymphocytes, monocytes, and eosinophils among all the groups studied.

\section{Discussion}

S. aureus is a human pathogen causing significant morbidity and mortality in both community- and hospitalacquired infections. Surprisingly, $S$. aureus frequently overcomes both host defense mechanisms and most antibiotic treatments. It is often found in chronic and recurrent infections and could be responsible for such infections. This bacterium is believed to have developed several means to combat conventional antibiotic therapies, among which are its ability to survive for long periods of time inside host cells (Garzoni and Kelley, 2009). The purpose of the present study was to examine whether intracellular $S$. aureus contributes to infection in vivo. Efforts were implemented to completely eradicate extra-cellular $S$. aureus in the inoculums by treating them with gentamicin; the complete killing of extra-cellular $S$. aureus was proved in our studies as well as in studies by others (Plotkowski et al., 1999; Hamrick et al., 2003; Hamad et al., 2010; Hamza and Li, 2012). Also, gentamicin remaining in the inoculums was expected to eliminate any extra-cellular $S$. aureus that leaked out within the first few hours.

We investigated whether intra-cellular S. aureus within osteoblasts could induce infection in our open femur fracture rat model. Note that this model is not an animal model of recurrent infection. This model was chosen because open fractures have high rates of infection and infection recurrence; a high rate of recurrent infections was reported in traumatic extremity injuries (Murray et al., 2008; Murray et al., 2011). We found that animals inoculated with $S$. aureus infected osteoblasts with intracellular S. aureus CFU of $10^{2}$ (i.e. Group IV) and $10^{6}$ (i.e. Group V) all led to severe infections, confirmed by culturing bone and muscle tissue from the infection sites and explanted K-wires (Fig. 3) and radiographic analysis of the fracture (Fig. 4). Inoculation of intra-cellular S. aureus alone of $10^{2} \mathrm{CFU}$ led to the same degree of infection as $10^{6}$
CFU inoculum, evidenced by approximately the same CFU in bone and muscle tissue at post-operative day 21 (Fig. 3 ). Animals inoculated with no bacteria (i.e. Groups I and II) or with $20 \mathrm{CFU}$ of extra-cellular $S$. aureus (i.e. Group III) did not have evidence of infection at post-operative day 21. No systemic infection was observed in any of the groups investigated because no CFU was found in blood samples. These results suggest that intra-cellular $S$. aureus inoculum of as low as $10^{2} \mathrm{CFU}$ within osteoblasts induced bone infections in our rat open femur fracture model and that the infection was localized.

It was interesting that severe bone infections were induced by intra-cellular $S$. aureus within osteoblasts at $10^{2} \mathrm{CFU}$, which was the same quantity of inoculum as our previous rat studies where $10^{2} \mathrm{CFU}$ of extra-cellular $S$. aureus was sufficient to induce bone infections in our open fracture rat model (Li et al., 2009; Li et al., 2010; Boyce et al., 2012). This suggested that not only extracellular but also intra-cellular $S$. aureus alone at $10^{2} \mathrm{CFU}$ could induce severe bone infections in open fractures. Note that inoculation of uninfected osteoblasts did not cause infection; no CFU was observed in corresponding bone, muscle, or K-wire samples (Fig. 3).

Meanwhile, neutrophils were significantly increased at post-operative day 21 in the groups inoculated with infected osteoblasts. This might suggest that neutrophils have played a deleterious role in infections induced by $S$. aureus infected osteoblasts. In the literature, neutrophils may have a protective or a deleterious role in $S$. aureus infection. On the one hand, neutrophils have long been believed to provide significant host defense against $S$. aureus infection. This is mainly because patients who are neutropenic or who have defects in neutrophil functions are more susceptible to $S$. aureus infection; total ablation of neutrophils in an experimental mouse model of $S$. aureus-induced septic arthritis led to decreased survival of the animals and increased bacteremia (Verdrengh and Tarkowski, 1997). On the other hand, increased neutrophils were also found to correlate with decreased survival of mice and increased bacterial burden at the infection site (Lowrance et al., 1994; Caver et al., 1996).

It is important to note that both in vitro and in vivo studies have shown that bacteria like $S$. aureus and $S$. epidermidis can internalize and survive within both phagocytic and non-phagocytic cells. In vitro, S. aureus can invade and survive within polymorphonuclear neutrophils and monocytes (Rogers and Tompsett, 1952; Kapral and Shayegani, 1959; Melly et al., 1960), endothelial cells (Vriesema et al., 2000), and osteoblasts (Hudson et al., 1995; Jevon et al., 1999; Ellington et al., 2003). Infected human osteoblasts were shown to release viable $S$. aureus, which was able to re-infect new osteoblasts (Ellington et al., 2003), while in this study the viability of infected osteoblasts decreased slightly over time $(84.6 \pm 6.7 \%$ at post-infection day 1 vs. $77.4 \pm 0.6 \%$ at day 7$)$. S. aureus can also internalize and perhaps even replicate within bovine mammary epithelial cells, both in cell lines and in primary culture (Almeida et al., 1996; Bayles et al., 1998). In vivo, intra-cellular $S$. aureus and $S$. epidermidis were found, in a few limited cases, in animals and patients. 
In animal studies, $S$. epidermidis was found to persist in macrophages in mice and may play an important role in the pathogenesis of biomaterial-associated infection (Boelens et al., 2000; Broekhuizen et al., 2008). S. aureus was found to be able to internalize and survive in osteoblasts of chick embryos (Reilly et al., 2000) and in bovine alveolar cells isolated from milk of infected cows (Hébert et al., 2000). More interestingly, $S$. aureus $\left(\sim 1 \times 10^{6} \mathrm{CFU}\right)$ surviving in neutrophils was able to establish infection after being injected intraperitoneally into naïve mice (Gresham et al., 2000). In human studies, intra-cellular $S$. aureus was found inside epithelium, glandular and myofibroblastic cells in intranasal biopsy specimens obtained, during infection symptom-free periods, from three patients with recurrent rhinosinusitis (Clement et al., 2005). Molecular typing proved the clonal relationship of the patientspecific Staphylococci. Intra-cellular S. aureus was also found in surgical bone specimens from a patient who had chronic, recurrent bone infection (Bosse et al., 2005). The intra-cellular $S$. aureus appeared to be sequestered and protected from the host immune system within the osteoblast or osteocyte cytoplasm for years (Bosse et al., 2005). In another case, intra-cellular S. aureus was cultured from five patients with persistent recurring osteomyelitis infections (Proctor et al., 1995). Alarmingly, some of the intra-cellular $S$. aureus were small-colony variants which were nonhemolytic and nonpigmented, grew very slowly on routine culture media, and were resistant to aminoglycosides under routine conditions.

Therefore, there is some in vitro and in vivo evidence supporting the potential role of intra-cellular $S$. aureus in Staphylococci pathogenesis. The results presented here are important because they demonstrate that intra-cellular S. aureus can induce infection in vivo. Bacteria (e.g. S. aureus) could internalize and survive within host cells (e.g. osteoblasts) and, after "hiding" for some time period (could be up to years, see Bosse et al., 2005), these quiescent bacteria could escape from the infected host cells into the surrounding tissue and could be sufficient to initiate a recurrence of symptomatic infection. It is also possible that, upon escaping from the host cells, bacteria may internalize into other host cells (e.g. neutrophils, macrophages). All of these may help to understand the pathogenesis of infections like periprosthetic joint infection, with which high infection recurrence (8-11\%) has been reported (Lieberman et al., 1994; Takahira et al., 2003) even after extensive antibiotic treatments during the two-stage exchange arthroplasty (gold standard in North America).

Taken together, our findings present direct in vivo evidence that intra-cellular $S$. aureus could induce bone infections and could be responsible for chronic and recurrent infections. These findings also suggested that as low as $10^{2} \mathrm{CFU}$ of intra-cellular $S$. aureus could induce severe bone infections in open fractures. These findings may greatly advance our understanding of the pathogenesis of bone infections, especially for chronic and recurrent infections. The induction of bone infections by intracellular $S$. aureus was studied in this work; however, the findings are expected to be applicable to other infections/ diseases and intra-cellular pathogens.

\section{Acknowledgements}

The authors acknowledge the surgical technical assistance from John Tidwell, MD and Alvin Jones, MD, and statistical assistance from Gerald R. Hobbs, PhD at West Virginia University. The authors also acknowledge financial support from the Osteosynthesis and Trauma Care Foundation, NSF (\#1003907), WV NASA EPSCoR, and WVU Health Sciences Center. The authors appreciate the use of the Flow Cytometry Core Facility at the West Virginia University Health Sciences Center. Microscope experiments and image analysis were performed in part in the West Virginia University Imaging Facility, which is supported in part by the Mary Babb Randolph Cancer Center and NIH grant P20 RR016440. D. P. also thanks the E. J. Van Liere fellowship for research. We wish to confirm that there are no known conflicts of interest associated with this publication and there has been no significant financial support for this work that could have influenced its outcome.

\section{References}

Almeida RA, Matthews KR, Cifrian E, Guidry AJ, Oliver SP (1996) Staphylococcus aureus invasion of bovine mammary epithelial cells. J Dairy Sci 79: 1021-1026.

Bayles KW, Wesson CA, Liou LE, Fox LK, Bohach GA, Trumble WR (1998) Intracellular Staphylococcus aureus escapes the endosome and induces apoptosis in epithelial cells. Infect Immun 66: 336-342.

Berendt T, Byren I (2004) Bone and joint infection. Clin Med 4: 510-518.

Boelens JJ, Dankert J, Murk JL, Weening JJ, van der Poll T, Dingemans KP, Koole L, Laman JD, Zaat SA (2000) Biomaterial-associated persistence of Staphylococcus epidermidis in pericatheter macrophages. J Infect Dis 181: 1337-1349.

Bosse MJ, Gruber HE, Ramp WK (2005) Internalization of bacteria by osteoblasts in a patient with recurrent, longterm osteomyelitis: A case report. J Bone Joint Surg Am 87: 1343-1347.

Boyce BM, Lindsey BA, Clovis NB, Smith ES, Hobbs GR, Hubbard DF, Emery SE, Barnett JB, Li B (2012) Additive effects of exogenous IL-12 supplementation and antibiotic treatment in infection prophylaxis. J Orthop Res 30: 196-202.

Broekhuizen CA, de Boer L, Schipper K, Jones CD, Quadir S, Vandenbroucke-Grauls CM, Zaat SA (2008) Staphylococcus epidermidis is cleared from biomaterial implants but persists in peri-implant tissue in mice despite rifampicin/vancomycin treatment. J Biomed Mater Res A 85: 498-505.

Canale ST (2003) Campbell's Operative Orthopaedics 10th ed. Mosby, St Louis, MO, pp 198-208.

Caver TE, O’Sullivan FX, Gold LI, Gresham HD (1996) Intracellular demonstration of active TGFbeta1 in B cells and plasma cells of autoimmune mice: IgG-bound TGFbeta1 suppresses neutrophil function and host defense against Staphylococcus aureus infection. J Clin Invest 98: 2496-2506. 
Ciampolini J, Harding KG (2000) Pathophysiology of chronic bacterial osteomyelitis: Why do antibiotics fail so often? Postgrad Med J 76: 479-483.

Clarke SR, Foster SJ (2006) Surface adhesins of Staphylococcus aureus. Adv Microb Physiol 51: 187-224.

Clement S, Vaudaux P, Francois P, Schrenzel J, Huggler E, Kampf S, Chaponnier C, Lew D, Lacroix JS (2005) Evidence of an intracellular reservoir in the nasal mucosa of patients with recurrent Staphylococcus aureus rhinosinusitis. J Infect Dis 192: 1023-1028.

de Lissovoy G, Fraeman K, Hutchins V, Murphy D, Song D, Vaughn BB (2009) Surgical site infection: incidence and impact on hospital utilization and treatment costs. Am J Infect Control 37: 387-397.

Easmon CS, Lanyon H, Cole PJ (1978) Use of lysostaphin to remove cell-adherent staphylococci during in vitro assays of phagocyte function. Br J Exp Pathol 59: 381-385.

Ellington JK, Harris M, Webb L, Smith B, Smith T, Tan K, Hudson M (2003) Intracellular Staphylococcus aureus: A mechanism for the indolence of osteomyelitis. J Bone Joint Surg Br 85: 918-921.

Garzoni C, Kelley WL (2009) Staphylococcus aureus: new evidence for intracellular persistence. Trends Microbiol 17: 59-65.

Gresham HD, Lowrance JH, Caver TE, Wilson BS, Cheung AL, Lindberg FP (2000) Survival of Staphylococcus aureus inside neutrophils contributes to infection. J Immunol 164: 3713-3722.

Hamad MA, Skeldon AM, Valvano MA (2010) Construction of aminoglycoside-sensitive Burkholderia cenocepacia strains for use in studies of intracellular bacteria with the gentamicin protection assay. Appl Environ Microbiol 76: 3170-3176.

Hamrick TS, Diaz AH, Havell EA, Horton JR, Orndorff PE (2003) Influence of extracellular bactericidal agents on bacteria within macrophages. Infect Immun 71: 1016-1019.

Hamza T, Li B (2012) Staphylococcus aureus internalization and associated osteoblast responses. 2012 Meeting of the Orthopaedic Research Society, San Francisco, CA, USA, paper 919.

Hébert A, Sayasith K, Sénéchal S, Dubreuil P, Lagacé J (2000) Demonstration of intracellular Staphylococcus aureus in bovine mastitis alveolar cells and macrophages isolated from naturally infected cow milk. FEMS Microbiol Lett 193: 57-62.

Hudson MC, Ramp WK, Nicholson NC, Williams AS, Nousiainen MT (1995) Internalization of Staphylococcus aureus by cultured osteoblasts. Microb Pathog 19: 409419.

Jevon M, Guo C, Ma B, Mordan N, Nair SP, Harris M, Henderson B, Bentley G, Meghji S (1999) Mechanisms of internalization of Staphylococcus aureus by cultured human osteoblasts. Infect Immun 67: 2677-2681.

Johnson EN, Burns TC, Hayda RA, Hospenthal DR, Murray CK (2007) Infectious complications of open type III tibial fractures among combat casualties. Clin Infect Dis 45: 409-415.
Kapral FA, Shayegani MG (1959) Intracellular survival of staphylococci. J Exp Med 110: 123-138.

Kumar JK (2008) Lysostaphin: an antistaphylococcal agent. Appl Microbiol Biotechnol 80: 555-561.

Li B, Jiang B, Boyce BM, Lindsey BA (2009) Multilayer polypeptide nanoscale coatings incorporating IL-12 for the prevention of biomedical device-associated infections. Biomaterials 30: 2552-2558.

Li B, Jiang B, Dietz MJ, Smith ES, Clovis NB, Rao KMK (2010) Evaluation of local MCP-1 and IL-12 nanocoatings for infection prevention in open fractures. $\mathrm{J}$ Orthop Res 28: 48-54.

Lieberman JR, Callaway GH, Salvati EA, Pellicci PM, Brause BD (1994) Treatment of the infected total hip arthroplasty with a two-stage reimplantation protocol. Clin Orthop Relat Res 301: 205-212.

Lowrance JH, O'Sullivan FX, Caver TE, Waegell W, Gresham HD (1994) Spontaneous elaboration of transforming growth factor beta suppresses host defense against bacterial infection in autoimmune MRL/lpr mice. J Exp Med 180: 1693-1703.

Maurin M, Raoult D (2001) Use of aminoglycosides in treatment of infections due to intracellular bacteria. Antimicrob Agents Chemother 45: 2977-2986.

Melly MA, Thomison JB, Rogers DE (1960) Fate of staphylococci within human leukocytes. J Exp Med 112: 1121-1130.

Murray CK, Hsu JR, Solomkin JS, Keeling JJ, Andersen RC, Ficke JR, Calhoun JH (2008) Prevention and management of infections associated with combat-related extremity injuries. J Trauma 64 (Suppl): S239-S251.

Murray CK, Obremskey WT, Hsu JR, Andersen RC, Calhoun JH, Clasper JC, Whitman TJ, Curry TK, Fleming ME, Wenke JC, Ficke JR (2011) Prevention of infections associated with combat-related extremity injuries. J Trauma 71 (Suppl): S235-S257.

Patti JM, Höök M (1994) Microbial adhesins recognizing extracellular matrix macromolecules. Curr Opin Cell Biol 6: 752-758.

Plotkowski MC, de Bentzmann S, Pereira SH, Zahm JM, Bajolet-Laudinat O, Roger P, Puchelle E (1999) Pseudomonas aeruginosa internalization by human epithelial respiratory cells depends on cell differentiation, polarity, and junctional complex integrity. Am J Respir Cell Mol Biol 20: 880-890.

Proctor RA, van Langevelde P, Kristjansson M, Maslow JN, Arbeit RD (1995) Persistent and relapsing infections associated with small-colony variants of Staphylococcus aureus. Clin Infect Dis 20: 95-102.

Pull ter Gunne AF, Cohen DB (2009) Incidence, prevalence, and analysis of risk factors for surgical site infection following adult spinal surgery. Spine 34: 14221428.

Reilly SS, Hudson MC, Kellam JF, Ramp WK (2000) In vivo internalization of Staphylococcus aureus by embryonic chick osteoblasts. Bone 26: 63-70.

Rogers DE, Tompsett R (1952) The survival of staphylococci within human leukocytes. J Exp Med 95: 209-230. 
Sax H, Lew D (1999) Osteomyelitis. Curr Infect Dis Rep 1: 261-266.

Sugarman B, Young EJ (1989) Infections associated with prosthetic devices: magnitude of the problem. Infect Dis Clin North Am 3: 1871-1898.

Takahira N, Itoman M, Higashi K, Uchiyama K, Miyabe M, Naruse K (2003) Treatment outcome of two-stage revision total hip arthroplasty for infected hip arthroplasty using antibiotic-impregnated cement spacer. J Orthop Sci 8: 26-31.

Verdrengh M, Tarkowski A (1997) Role of neutrophils in experimental septicemia and septic arthritis induced by Staphylococcus aureus. Infect Immun 65: 2517-2521.

Vriesema AJ, Beekhuizen H, Hamdi M, Soufan A, Lammers A, Willekens B, Bakker O, Welten AG, Veltrop MH, van De Gevel JS, Dankert J, Zaat SA (2000) Altered gene expression in Staphylococcus aureus upon interaction with human endothelial cells. Infect Immun 68: 1765-1772.

Wright KM, Friedland JS (2002) Differential regulation of chemokine secretion in tuberculous and staphylococcal osteomyelitis. J Bone Miner Res 17: 1680-1690.

Zalavras CG, Patzakis MJ (2003) Open fractures: evaluation and management. J Amer Acad Orthop Surg 11: 212-219.

\section{Discussion with Reviewer}

Reviewer I: The authors state that $10^{2}$ and $10^{6} \mathrm{~S}$. aureus caused infection in earlier studies when added as free bacteria, and therefore selected $20 \mathrm{CFU}$ as the number of CFU to administer as free bacteria control in this study. The lowest dose of bacteria administered as internalized bacteria in osteoblasts, however, was $100 \mathrm{CFU}$. It might have been more systematic to use the same number of bacteria as free inoculum and as internalized inoculum, i.e. also have a group infected with only 20 CFU internalized. Can the authors please comment on this?

Authors: This reviewer makes a good point. From our previous studies, we knew that $10^{2} \mathrm{CFU} / 0.1 \mathrm{~mL}$ of extracellular $S$. aureus induced infection in our open fracture rat model (Li et al., 2009; Li et al., 2010; Boyce et al., 2012). We hoped that $20-50 \mathrm{CFU} / 0.1 \mathrm{~mL}$ would not induce infection and the inclusion of such a group of low extracellular $S$. aureus would help us evaluate the potential role of intra-cellular $S$. aureus in inducing infection by comparing Group III with Group VI in this study. It would be another interesting study to have a group infected with only $20 \mathrm{CFU}$ of intra-cellular $S$. aureus to determine if such a group will result in infection as well. If such a group would result in infection while $20 \mathrm{CFU}$ of extra-cellular S. aureus did not lead to infection as found in this study, it would mean that intra-cellular pathogens are more virulent. 